\title{
Detection of gamma-hydroxybutyrate in hair: Validation of GC-MS and LC-MS/MS methods and application to a real case
}

\author{
Elisabetta Bertol ${ }^{\mathrm{a}, *}$, Antonina Argo ${ }^{\mathrm{b}}$, Paolo Procaccianti ${ }^{\mathrm{b}}$, Fabio Vaiano ${ }^{\mathrm{a}}$, Maria Grazia Di Milia ${ }^{\mathrm{a}}$, \\ Sandra Furlanetto ${ }^{c}$, Francesco Mari ${ }^{a}$ \\ a Forensic Toxicology Division, Institute of Legal Medicine, University of Florence, Italy \\ ${ }^{\mathrm{b}}$ Department of Biopathology, Biotechnology and Legal Medicine, University of Palermo, Italy \\ ${ }^{\mathrm{c}}$ Department of Pharmaceutical Sciences, University of Florence, Italy
}

\section{A R T I C L E I N F O}

\section{Article history:}

Received 21 May 2012

Received in revised form 10 July 2012

Accepted 12 July 2012

Available online 25 July 2012

\section{Keywords:}

Gamma-hydroxybutyrate

GHB

Hair

Segmental analysis

GC-MS

LC-MS/MS

\begin{abstract}
A B S T R A C T
A gas chromatography-mass spectrometry (GC-MS) and a liquid chromatography tandem mass spectrometry (LC-MS/MS) method were validated for quantifying endogenous and exogenous hair concentrations of gamma-hydroxybutyrate (GHB). The GC-MS method is based on overnight extraction of $25 \mathrm{mg}$ hair in $\mathrm{NaOH}$ at $56^{\circ} \mathrm{C}$, liquid/liquid extraction in ethylacetate and trimethylsylil derivatization; analysis is by electron ionization and single ion monitoring of three ions. The LC-MS/MS method entails a rapid digestion of $25 \mathrm{mg}$ hair with $\mathrm{NaOH}$ at $75^{\circ} \mathrm{C}$ for $40 \mathrm{~min}$, liquid/liquid extraction in ethylacetate and reconstitution of the extract in the LC mobile phase; negative ion electrospray ionization and multiple reaction monitoring (MRM) analysis are employed for the LC-MS/MS detection. In both cases, GHB-d6 is used as an internal standard. The endogenous amount in "blank" hair are estimated by the standard addition method. Limits of detection are 0.4 and $0.5 \mathrm{ng} / \mathrm{mg}$ for GC-MS and LC-MS/MS respectively, while the limit of quantification (LOQ) is $0.6 \mathrm{ng} / \mathrm{mg}$ for both methods; the GC-MS method proved to be linear in the range $1-50 \mathrm{ng} / \mathrm{mg}$ whereas linearity was demonstrated from 0.6 to $50 \mathrm{ng} / \mathrm{mg}$ for the LC-MS/MS; imprecision and inaccuracy were always lower than $23 \%$ for quality controls samples. The two methods were applied to a real case of a man addicted to GHB; the drug concentration in segments from $17 \mathrm{~cm}$ hair strand well correlated with self-reported use of GHB in different periods of his life. Performances of the two methods were similar.
\end{abstract}

(C) 2012 Elsevier B.V. All rights reserved.

\section{Introduction}

Gamma-hydroxybutyrate (GHB), due to the endogenous metabolism of gamma-aminobutyric acid (GABA), is present in small concentration in different biological samples [1]. In forensic cases, differentiation between endogenous and exogenous GHB is of great importance [2-7]. As per the Italian law, GHB is a schedule I central nervous system depressant drug, producing euphoria, relaxation, respiratory depression at moderate doses, or coma and death at higher doses. GHB is also a pharmaceutical, prescription drug (schedule II in Italy, III in USA), as Xiram ${ }^{\circledR}$ and Alcover ${ }^{\circledR}$, used in the clinical treatment of cataplexy or narcolepsy, alcohol abuse, and, recently, in the trial treatment of Alzheimer's cases [8]. Illicit use of GHB is common among young people, especially during rave parties and among body builders. Use of GHB as recreational drug is well known as causing driving impairment $[9,10]$. More recently GHB has also been used as a drug facilitating sexual assault (DFSA)

\footnotetext{
* Corresponding author. Tel.: +39 055 7947208-6178; fax: +39 0557946171

E-mail address: elisabetta.bertol@unifi.it (E. Bertol).
}

or as a "date-rape" drug $[11,12]$. It is easily available on the street market, in fitness or dancing clubs or in the Internet, as well as its precursor gamma-butyrolactone (GBL). The purpose of this study was the development and validation of qualitative and quantitative procedures to detect GHB in hair by using and comparing a more traditional, gas chromatography-mass spectrometric method (GC-MS) and a liquid chromatography-tandem mass spectrometry (LC-MS/MS) one. The two methods were developed in hair to document previous use of GHB either in chronic use or single exposure (i.e. sexual assault). Both methods must take into account the physiological concentrations of GHB in human hair, which is an important caveat in forensic cases [12-15]. The use of hair as a matrix proves particularly valuable when GHB administration is referred or suspected at a later time, as was in a case of a 24-year-old girl who was sexually assaulted [16].

\section{Materials and methods}

\subsection{Hair samples}

The hair of volunteers (laboratory personnel) was cut at the posterior-vertex region as close to the scalp as possible and stored 
at room temperature in a plastic vial. Hair was decontaminated twice using $5 \mathrm{~mL}$ of methylene chloride for $2 \mathrm{~min}$, dried, and cut into small fragments ( $\leq 1 \mathrm{~mm}$ ). Portions of $25 \mathrm{mg}$ were used for preparation of controls and calibrators, and $2 \mathrm{ng} / \mathrm{mg}$ of deuterated IS (GHB-d6) were added. Since endogenous GHB could interfere with the determination of exogenous concentrations, considering that no GHB free hair are in principle available, the standard addition method was used for calibration.

\subsection{Chemicals}

Water, methanol (MeOH), N,O-bis trimethylsilyltrifluoroacetamide (BSTFA) with $1 \%$ trimethylchlorosylane (TMCS) were purchased from Sigma-Aldrich (St. Louis, MO, USA). Sodium hydroxide $(\mathrm{NaOH})$, sulfuric acid $\left(\mathrm{H}_{2} \mathrm{SO}_{4}\right)$, formic acid and ethyl acetate were purchased from J.T. Baker (Deventen, Holland). GHB and GHB-d6 (internal standard, IS) were purchased from Chemical Research 2000 s.r.l. (Rome, Italy).

\subsection{GC-MS method}

\subsubsection{Extraction}

Decontaminated hair aliquots of $25 \mathrm{mg}$ were incubated overnight in $1.0 \mathrm{~mL}$ of $0.01 \mathrm{~N} \mathrm{NaOH}$, at $56^{\circ} \mathrm{C}$, in the presence of $50 \mathrm{ng}$ of GHB-d6 used as an internal standard (IS, $2 \mathrm{ng} / \mathrm{mg}$ hair). After cooling, the homogenate was neutralized with $0.1 \mathrm{~mL}$ of $\mathrm{H}_{2} \mathrm{SO}_{4} 0.1 \mathrm{~N}$; $3.0 \mathrm{~mL}$ of ethylacetate and $0.01 \mathrm{~mL}$ of $\mathrm{H}_{2} \mathrm{SO}_{4}$ were added. After agitation and centrifugation, the supernatant layer was evaporated to dryness and derivatized with $20 \mu \mathrm{L}$ BSTFA, $1 \%$ TMCS in $20 \mu \mathrm{L}$ ethyl acetate $\left(20 \mathrm{~min}\right.$ at $\left.60^{\circ} \mathrm{C}\right)$.

\subsubsection{Instrumental procedure}

The GC-MS instrument consisted in an Agilent 7890A GC system equipped with an Agilent 7683B series autosampler (Agilent Technologies, Palo Alto, CA, USA), and interfaced to a single quadrupole Agilent 5975C mass spectrometer (Agilent Technologies, Palo Alto, CA, USA). The column used was an Agilent HP-5MS, 30 m length, $0.25 \mathrm{~mm}$ i.d. and $0.25 \mu \mathrm{m}$ film thickness (Agilent Technologies, Palo Alto, CA, USA). The gas carrier (He) flow was constant at $1 \mathrm{~mL} / \mathrm{min}$. The oven temperature was set initially at $60^{\circ} \mathrm{C}$ for $0.5 \mathrm{~min}$, and programmed to $130^{\circ} \mathrm{C}$ at $10^{\circ} \mathrm{C} / \mathrm{min}$, and $300^{\circ} \mathrm{C}$ at $8^{\circ} \mathrm{C} / \mathrm{min}$. Injector and transfer line temperatures were respectively $300^{\circ} \mathrm{C}$ and $230^{\circ} \mathrm{C}$. Electron ionization and selected ion monitoring (SIM) acquisition mode were used and the ions of interest were 233, 234, 147 for GHB and 239, 240 and 147 for IS. $1 \mu \mathrm{L}$ of the derivatized extracts was injected in splitless mode.

\subsubsection{Method validation}

LOD (limit of detection) and LOQ (limit of quantification), defined as the lowest concentrations that produce signal/noise ratios values of at least 3 and 10 respectively, were calculated analyzing $25 \mathrm{mg}$ hair aliquots decontaminated and added with decreasing GHB amounts. Linearity was evaluated analyzing different calibration levels, and applying the least-square regression method to build the calibration curve. The concentration levels used were 1.0, 5.0, 10.0, 20.0 and $50.0 \mathrm{ng} / \mathrm{mg}$, and for each level 5 replicates were carried out. Accuracy and precision were evaluated using three quality controls (QC) at $1.5,2.5$ and $15 \mathrm{ng} / \mathrm{mg}$. Imprecision, as degree of repeatability, was estimated as the average of relative standard deviation (\%RSD) values calculated for QC samples with 5 replicates. The intra-day and inter-day imprecisions were calculated in the same day and in 5 different days during a month, respectively. As certified reference materials (CRM) were not available, accuracy (or trueness [17]) was evaluated as \%mean relative error (\%MRE) on the basis of the total data set $(n=25)$ at the above three concentrations. Recovery was estimated by comparing peak areas obtained by adding GHB and IS before and after the extraction, for 3 replicates at the concentration of 1.5, 2.5 and $15.0 \mathrm{ng} / \mathrm{mg} \mathrm{GHB}$ and $2 \mathrm{ng} / \mathrm{mg}$ IS.

\subsection{LC-MS/MS method}

\subsubsection{Extraction}

Portions of $25 \mathrm{mg}$ decontaminated hair were digested with $500 \mu \mathrm{L} 1 \mathrm{M} \mathrm{NaOH}$ in the presence of $50 \mathrm{ng}$ IS at $75^{\circ} \mathrm{C}$ for $40 \mathrm{~min}$. After cooling at room temperature, the sample was added with $600 \mu \mathrm{L}$ of $1 \mathrm{M} \mathrm{H}_{2} \mathrm{SO}_{4}$ and $3 \mathrm{~mL}$ of ethyl acetate, immediately agitated and centrifuged. The supernatant organic layer was dried and reconstituted in $50 \mu \mathrm{L}$ of mobile phase; $6 \mu \mathrm{L}$ was injected into the LC-MS/MS.

\subsubsection{Instrumental procedure}

Analysis was performed with an HPLC Agilent 1290 Infinity system (Agilent Technologies, Palo Alto, CA, USA), coupled to an Agilent 6460 Triple Quadrupole (Agilent Technologies), and equipped with an electrospray ion source (ESI). The column used was a Zorbax SB-C18 Rapid Resolution HT $(2.1 \mathrm{~mm} \times 50 \mathrm{~mm}, 1.8 \mu \mathrm{m}$, Agilent Technologies, Palo Alto, CA, USA), heated at $30^{\circ} \mathrm{C}$. Mobile phases consisted of $5 \mathrm{mM}$ aqueous formic acid (A) and methanol (B). Starting from $20 \% \mathrm{~B}$, gradient elution was carried out by increasing $B$ to $99 \%$ at $3 \mathrm{~min}$, then running isocratically for $1 \mathrm{~min}$; post-run time was $1.5 \mathrm{~min}$. The flow rate was $0.4 \mathrm{~mL} / \mathrm{min}$. Negative ion ESI configuration was: gas temperature $325^{\circ} \mathrm{C}$; gas flow rate $10 \mathrm{~L} / \mathrm{min}$; nebulizer 20 psi; capillary $4000 \mathrm{~V}$. Collisional experiments and multiple reaction monitoring (MRM) analysis were performed, with a fragmentor voltage of $75 \mathrm{~V}$ for GHB and IS; collision energies were $9 \mathrm{~V}$ for the transition $103>57$ ( $109>61$ for IS) and $5 \mathrm{~V}$ for the transition $103>85$ ( 109 > 90 for IS); a dwell time of 200 ms was used.

\subsubsection{Method validation}

The evaluating criteria were the same used for the above described GC-MS method. After a first evaluation, the calibrators were prepared at $0.6,1.0,5.0,10.0,20.0$ and $50.0 \mathrm{ng} / \mathrm{mg}$, and for each level 5 replicates were carried out. Imprecision and accuracy were evaluated, as described above for GC-MS, using QCs at 0.7, 2.3 and $10.3 \mathrm{ng} / \mathrm{mg}$, run in 5 replicates a day for 5 days in a month. Analogously to GC-MS method, recovery was estimated for 3 replicates at the concentration of $0.7,2.3$ and $10.3 \mathrm{ng} / \mathrm{mg}$ and $2 \mathrm{ng} / \mathrm{mg}$ IS..

\section{Results}

\subsection{Validation results}

By adding increasing amounts of the analyte to hair matrix free from exogenous GHB (donated by laboratory personnel) we were able to estimate the endogenous level by regression analysis of the calibration curve. We obtained an estimated concentration of $0.28 \mathrm{ng} / \mathrm{mg}$ with GC-MS, and 0.27 with LC-MS/MS, in the low end of the range reported in the literature [11-15]; we subsequently used the calibration curves obtained with that specific matrix to quantify QCs and samples, and corrected the amounts of found GHB by adding the value of the "blank." Obviously, when using the standard addition method, the GHB content of the blank must be recalculated in case calibration curves are prepared with fresh hair. LOD and LOQ of the GC-MS assay were $0.4 \mathrm{ng} / \mathrm{mg}$ and $0.60 \mathrm{ng} / \mathrm{mg}$, as determined empirically by fortifying $25 \mathrm{mg}$ aliquots of hair with decreasing quantities of GHB. After studying the linearity of the method by regression analysis, an average $R^{2}$ of $0.9997(n=5)$ could be obtained only when the lowest quantification point at $0.6 \mathrm{ng} / \mathrm{mg}$ was removed and the studied range was from 1.0 to $50.0 \mathrm{ng} / \mathrm{mg}$. 
Table 1

Validation parameters for GC-MS analysis.

\begin{tabular}{|c|c|c|c|c|c|}
\hline \multicolumn{6}{|c|}{ Intra-day $(n=5)$} \\
\hline $\mathrm{QC}, \mathrm{ng} / \mathrm{mg}$ & \multicolumn{2}{|l|}{ Average conc., ng/mg } & \multicolumn{2}{|c|}{ Average SD, ng/mg } & \%RSD \\
\hline 1.5 & 1.7 & & 0.31 & & 18 \\
\hline 2.5 & 2.7 & & 0.41 & & 15 \\
\hline 15.0 & 15.9 & & 0.98 & & 6 \\
\hline \multicolumn{6}{|c|}{ Inter-day $(n=25)$} \\
\hline $\mathrm{QC}, \mathrm{ng} / \mathrm{mg}$ & Average conc., ng/mg & Averag & ge $\mathrm{SD}, \mathrm{ng} / \mathrm{mg}$ & $\%$ RSD & $\% \mathrm{MRE}$ \\
\hline 1.5 & 1.6 & 0.37 & & 23 & 7 \\
\hline 2.5 & 2.7 & 0.40 & & 15 & 10 \\
\hline 15.0 & 14.4 & 1.17 & & 8 & 4 \\
\hline
\end{tabular}

This is not unusual, considering that the LOQ was determined by fortifying hair with decreasing quantities of GHB and not by constructing a dedicated curve at low concentration levels. In Table 1 are reported some validation parameters. As may be seen, imprecision was always smaller than $23 \%$ for all the QCs in both intra-day and inter-day evaluations, and inaccuracy, calculated as \%MRE of spiked samples since CRMs are not available, was acceptably low $(<10 \%)$. The extraction recoveries ranged from $89.5 \%$ (lowest QC) to 92.3\% (highest QC).

As to the LC-MS/MS method, its LOD and LOQ values were respectively 0.5 and $0.6 \mathrm{ng} / \mathrm{mg}$. Not withstanding a higher LOD, GHB response was linear from 0.6 to $50.0 \mathrm{ng} / \mathrm{mg}$, i.e. including the LOQ, with an average correlation coefficient of $0.9983(n=5)$. A possible explanation of the extended linear range of the LC-MS/MS method is the higher degree of specificity and lower level of interferences observed when working in MRM mode. In Table 2 are reported the data pertaining imprecision and inaccuracy. Intra-day imprecision was always lower than $19 \%$; inter-day values were better than $23 \%$; inaccuracy was $\leq 9 \%$. Recoveries ranged from $89.7 \%$ for QC at $0.67 \mathrm{ng} / \mathrm{mg}$ to $97.9 \%$ for QC at $10.3 \mathrm{ng} / \mathrm{mg}$. In Fig. 1, a MRM chromatogram analysis of hair containing GHB at $0.7 \mathrm{ng} / \mathrm{mg}$ is presented. As may be inferred, high signal-to-noise ratio is obtained and the chromatogram appears to be free from interferences at the retention time of the analyte.

It must be emphasized that the GC-MS method was adapted from a routine GC-MS method used in our laboratory for detection of acidic drugs in hair, entailing a night-long digestion of a relatively large amount of hair ( $25 \mathrm{mg}$ ) in basic conditions, neutralization and derivatization. On the contrary, the LC-MS/MS method profits of a rapid sequence of digestion ( $40 \mathrm{~min}$ ), extraction and redissolution of the extract in the LC mobile phase: time of preparation is therefore dramatically reduced as proposed by Kerrigan [5]. For validation and comparison purposes, the two methods were initially applied to $25 \mathrm{mg}$ hair, but work is in progress to reduce the sample amount, that would allow an easier application of the method for segmental analysis when tiny hair specimen are available.

Table 2

Validation parameters for LC-MS/MS method.

\begin{tabular}{lllc}
\hline \multicolumn{2}{l}{ Intra-day $(n=5)$} & & \\
\hline QC, ng/mg & Average conc., ng/mg & Average SD, ng/mg & \%RSD \\
\hline 0.7 & 0.75 & 0.14 & 19 \\
2.3 & 2.17 & 0.26 & 12 \\
10.3 & 11.4 & 0.45 & 4
\end{tabular}

Inter-day $(n=25)$

\begin{tabular}{cllrc}
\hline QC, ng/mg & Average conc., ng/mg & Average SD, ng/mg & \%RSD & \%MRE \\
\hline 0.7 & 0.72 & 0.17 & 23 & 7 \\
2.3 & 2.48 & 0.37 & 15 & 9 \\
10.3 & 9.37 & 0.66 & 7 & 9 \\
\hline
\end{tabular}

Table 3

Results from case hair sample.

\begin{tabular}{rccc}
\hline Segment \# & $\begin{array}{l}\text { Distance from } \\
\text { the root }(\mathrm{cm})\end{array}$ & GHB concentration $(\mathrm{ng} / \mathrm{mg})$ \\
\cline { 3 - 4 } & & GC-MS method & LC-MS/MS method \\
\hline 1 & 1 & 3.52 & 4.08 \\
2 & 2 & 1.49 & 1.26 \\
3 & 3 & 1.20 & 1.38 \\
4 & 5 & 4.74 & 4.56 \\
5 & 7 & 7.97 & 6.48 \\
6 & 9 & 8.93 & 7.23 \\
7 & 11 & 9.53 & 8.56 \\
8 & 13 & 8.65 & 7.39 \\
9 & 15 & 8.22 & 8.95 \\
10 & 17 & 10.34 & 9.15 \\
\hline
\end{tabular}

\subsection{Application to a real case}

A 40-year-old man was arrested by the Police during an investigation on the Internet commerce of gamma butyrolactone (GBL) an industrial solvent that can be used as a precursor of GHB and is also scheduled in Table 1 as prohibited substance. The man was persecuted for a drug crime having bought large quantities of GBL to prepare GHB and, purportedly, to sell it in the illicit market. His justification was based on a self-reported, long history of GHB abuse that made him addict to the drug and craving for it. His first contacts with GHB dated back to a therapeutic approach for alcohol abuse with Alcover. Following the therapy for years, he had become addict so that he had a compulsory behavior for GHB supply. Signs of craving and withdrawal symptoms were observed during a medico-legal visit permitted by the judge two months after his arrest, converted in "house arrest".

The subject consented to have a hair test to prove his history of abuse; he had long, brown hair (about $17 \mathrm{~cm}$, cut from the posterior vertex at the surface of the scalp), so it was possible to investigate over a life period approximately corresponding to 10-14 months, considering an average hair growth of $1.0-1.5 \mathrm{~cm} / \mathrm{month}$. In the 2 month period from his arrest to hair sampling, the subject under investigation had no contact with the drug, thereafter the GHB content in the $3 \mathrm{~cm}$ proximal hair segment reasonably represent his endogenous level. The hair samples were decontaminated and segmented in 10 fragments, the first three segments were $1 \mathrm{~cm}$ long, the others $2 \mathrm{~cm}$ long. The authors wanted to document in detail the most recent period of life of the man, and the $1 \mathrm{~cm}$ segmentation proved useful to detect a higher level just at the root, and two subsequently lower levels. If we had used a $3 \mathrm{~cm}$ long segment for the same period, as suggested by the literature, the two month at no exogenous GHB periods would have been confounded by the higher level of GHB at the root. On every segment, both methods (GC-MS and LC-MS/MS) were applied to $25 \mathrm{mg}$ aliquots. Results of hair analysis are shown in Table 3 whereas Fig. 2 shows the same GHB concentrations plotted vs the hair length or observation period, as calculated from length, with emphasis on the time of arrest (Fig. 3).

\section{Discussion}

Both methods were able to assay GHB concentrations at the endogenous level in hair, reported from the literature to be $\leq 2 \mathrm{ng} / \mathrm{mg}$. The LOQs were indeed analogous and the estimate of GHB physiological level in a blank matrix by the standard addition methods yielded similar responses; however, the LC-MS/MS method showed a wider linearity range than GC-MS. The GC-MS method proposed here is an adaptation of a method routinely 


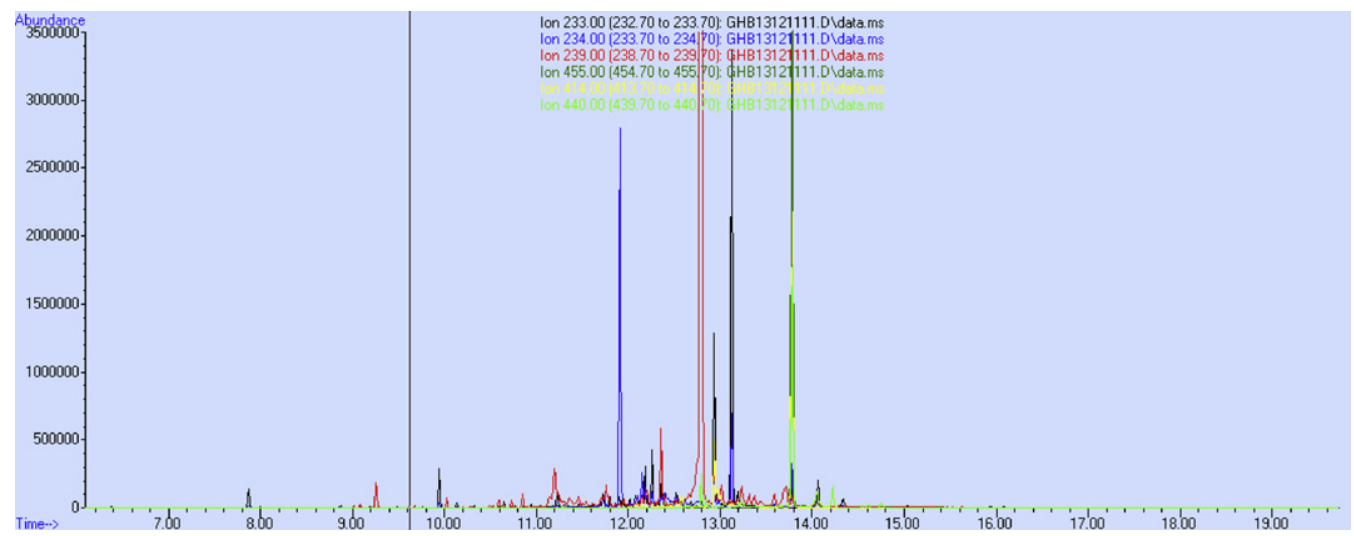

Fig. 1. SIM chromatogram for GHB and GHB-d6 in a QC spiked at $1.0 \mathrm{ng} / \mathrm{mg}$.
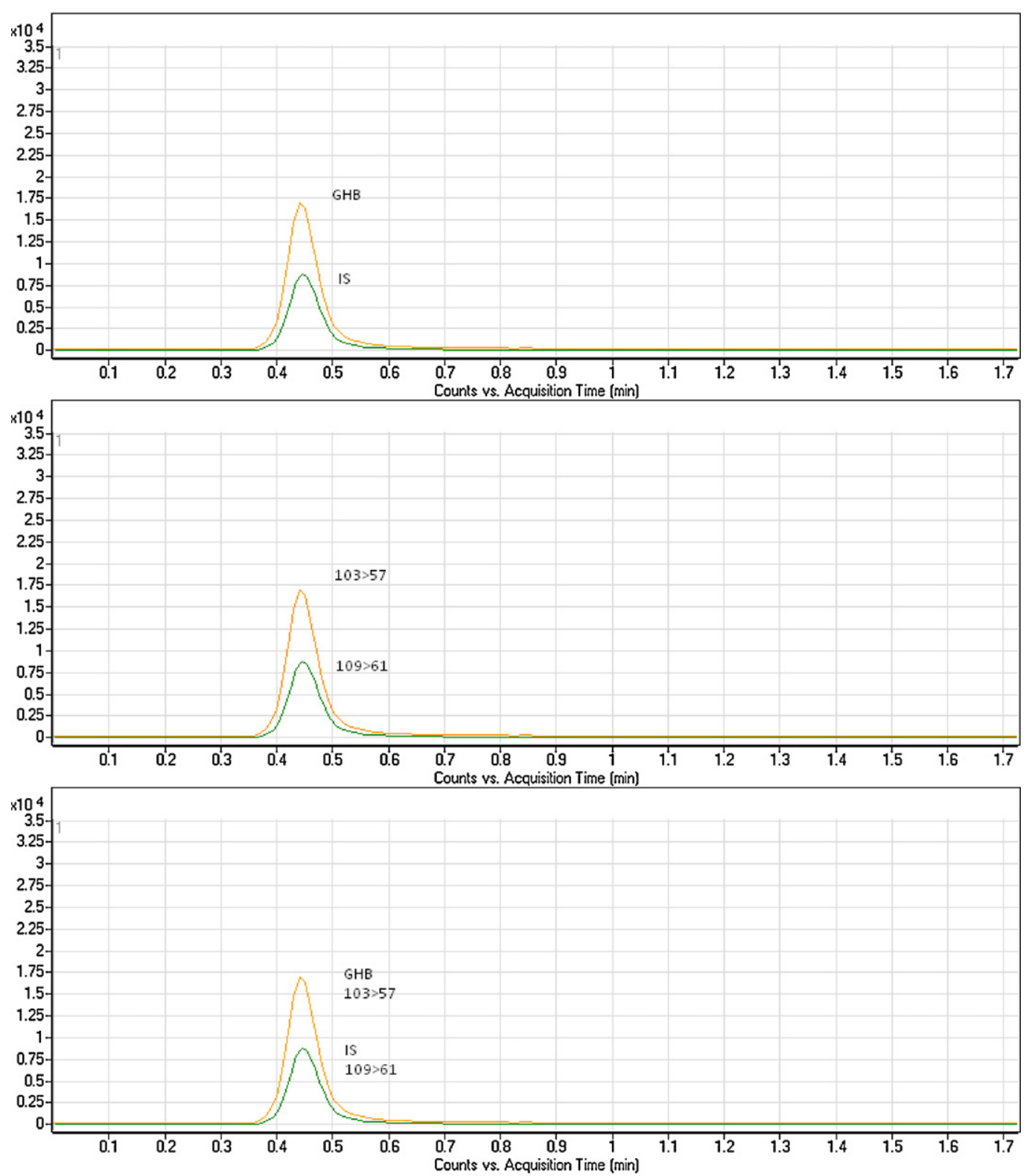

Fig. 2. MRM chromatogram for GHB and GHB-d6 in a $Q C$ spiked at $0.7 \mathrm{ng} / \mathrm{mg}$. 


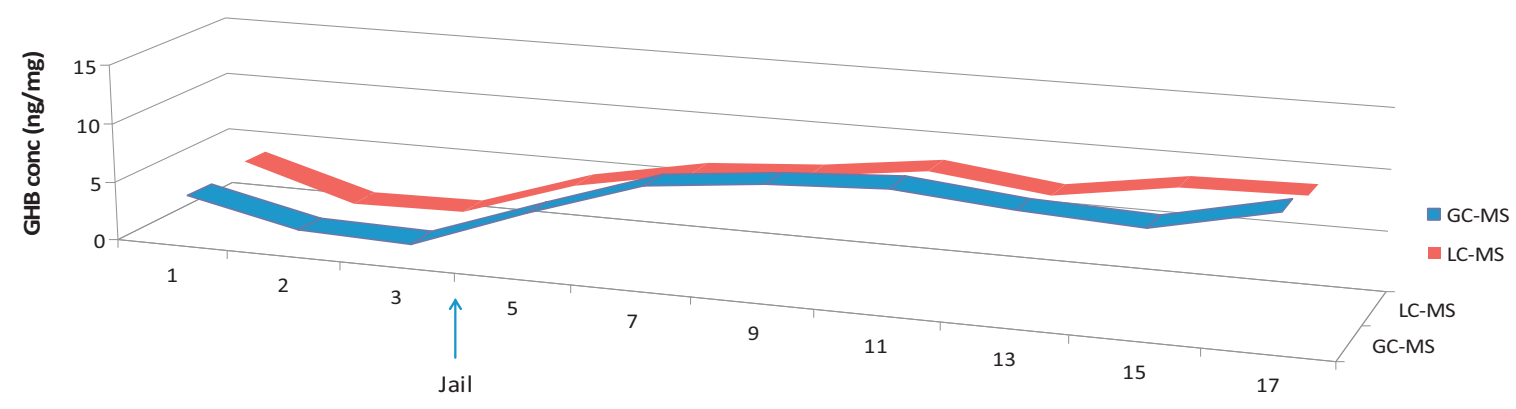

$\mathrm{cm}$ from the root (months)

Fig. 3. Hair segmentation from the case subject, with indication of his arrest, about two months before hair collection.

used for the analysis of acidic drugs in hair. It employs a single quadrupole of the latest generation (Agilent 5975) and its LOD of 0.4 and LOQ of $0.6 \mathrm{ng} / \mathrm{mg}$ in hair must be regarded as particularly valuable, since they afford to reliably identify GHB by the simplest and most widespread instrumentation in a toxicology laboratory, in particular when a history of chronic use or addiction must be investigated. However, the identification and documentation of a single exposure to the drug, when GHB is used as a rape drug in DFSA, when accuracy is required at low concentration levels, may take advantage of the more specific and selective LC-MS/MS method. As to the pre-analytical step, it must be noticed that hair sample extraction for LC-MS/MS analysis, as proposed by Kerrigan et al. [7], is much more faster than for GC-MS, but a reduction in hair sample size is required.

In order to directly compare the efficiency of both methods, we applied them to the same hair sample, obtained from a case in which the use of GHB was documented by a past history of treatment with Alcover and addiction. The quantitative data obtained are really similar, even if the LC-MS/MS appears to moderately underestimate the GHB content; the general trend is fairly similar, and analogous information can be inferred, useful to the forensic toxicologist. As to the real case, our results clearly demonstrated a neat decreasing of GHB concentration in the period corresponding to arrest and house custody. The very low concentrations detected in the 2nd and $3 \mathrm{rd} \mathrm{cm}$ from the scalp well represent the physiological GHB level of the subject, in agreement with the normal level observed in human hair $(\leq 2 \mathrm{ng} / \mathrm{mg}$ ). The quite higher concentration observed in the first segment was reasonably due to the endogenous GHB incorporation through sweat at the root level, as reported in the literature $[11,14]$.

\section{Conclusions}

In conclusion, a GC-MS and a LC-MS/MS method were validated, compared and applied to the detection and quantification of GHB in $25 \mathrm{mg}$ hair. They both proved to be suitable for the determination of the drug in cases of chronic use, and to discriminate between the endogenous and the exogenous GHB levels, exhibiting LOQs of $0.6 \mathrm{ng} / \mathrm{mg}$. Their application to the analysis of hair segments in a real case of GHB addiction yielded comparable results that were useful to disclose the pattern of use/abuse of the investigated man.
In order to corroborate the efficacy of the more specific LC-MS/MS method in single exposure (i.e. in DFSA) other real cases, with these characteristics will be included in future applications.

\section{References}

[1] L. Tedeschi, M.A. Carai, G. Frison, D. Favretto, G. Colombo, S.D. Ferrara, G.L Gessa, Endogenous gamma-hydroxybutyric acid is in the rat, mouse and human gastrointestinal tract, Life Sci. 72 (2003) 2481-2488.

[2] N. Shima, A. Miki, T. Kamata, M. Katagi, H. Tsuchihasi, Endogenous level and in vitro production of GHB in blood from healthy humans and the interpretation of GHB levels detected in ante-mortem blood samples, J. Health Sci. 51 (2005) $147-154$.

[3] E. Kaufmann, A. Alt, Determination of GHB in urine and serum by LC-MS using a simple one-step derivative, Forensic Sci. Int. 3 (2007) 133-137.

[4] M.A. Le Beau, R.H. Christenson, B. Levine, D.W. Darwin, M.A. Huestis, Intra-and inter-individual variations in urinary concentrations of endogenous gammahydroxybutyrate, J. Anal. Toxicol. 26 (2002) 340-346.

[5] S. Kerrigan, In vitro production of gamma-hydroxybutyrate in antemortem urine samples, J. Anal. Toxicol. 26 (2002) 571-574

[6] F. Mari, L. Politi, C. Trignano, M.G. Di Milia, M. Di Padua, E. Bertol, What constitutes a normal ante-mortem urine GHB concentration? J. Forensic Legal Med 1 (2008) 1-3.

[7] P.A. Stout, M.S. Kelsie, D. Simons, S. Kerrigan, Quantitative analysis of gamma-hydroxybutyrate at endogenous concentrations in hair using liquid chromatography tandem mass spectrometry, J. Forensic Sci. 55 (2010) 531-537.

[8] M. Mamelak, Alzheimer' s disease, oxidative stress and gammahydroxybutyrate, Neurobiol. Aging 28 (2007) 1340-1360.

[9] A.W. Jones, A. Holmgren, F.C. Kugelberg, Driving under the influence of gammahydroxybutyrate (GHB), Forensic Sci. Med. Pathol. 4 (2008) 205-211.

[10] F.J. Couper, B.K. Logan, GHB and driving impairment, J. Forensic Sci. 46 (2001) 919-923.

[11] P. Kintz, V. Cirimele, C. Jamey, B. Ludes, Testing for GHB in hair by GC-MS/MS after a single exposure. Application to document sexual assault, J. Forensic Sci. 48 (2003) 195-200.

[12] D. Wells, Drug administration and sexual assault: sex in the glass, Sci. Justice 41 (2001) 197-199.

[13] P. Kintz, M. Villain, A.L. Pélissier, V. Cirimele, Unusually high concentrations in a fatal GHB case, J. Anal. Toxicol. 29 (2005) 582-585.

[14] J.P. Goullé, M. Chèze, G. Pépin, Determination of endogenous levels of GHB in human hair: are there possibilities for the identification of GHB administration through hair analysis in cases of drug-facilitated sexual assault? J. Anal. Toxicol. 27 (2003) 574-580.

[15] G. Pepin, Analytical, toxicological and forensic aspects of drug-facilitated crimes: 10 years of experience, Ann. Pharm. Fr. 68 (2010) 61-75.

[16] R. Rossi, M. Lancia, C. Gambelunghe, A. Oliva, N. Fucci, Identification of GHB and morphine in hair in a case of drug-facilitated sexual assault, Forensic Sci. Int. 186 (2009) e9-e11.

[17] ISO 5725-1:1994. Accuracy (trueness and precision) of Measurement Methods and Results. Part 1: General Principles and Definitions. 